\title{
A Simple Method for Forming Hybrid Core-Shell Nanoparticles Suspended in Water
}

\author{
Jean-Christophe Daigle and Jerome P. Claverie \\ NanoQAM-Research Center for Functional Materials, University of Québec at Montréal, Succursale Centre Ville,
} Montreal, Québec, Canada H3C3P8

Correspondence should be addressed to Jerome P. Claverie, claverie.jerome@uqam.ca

Received 21 March 2008; Accepted 9 July 2008

Recommended by Claude Estournes

Core-shell hybrid nanoparticles, where the core is an inorganic nanoparticle and the shell an organic polymer, are prepared by a two-step method. Inorganic nanoparticles are first dispersed in water using poly(acrylic acid) (PAA) prepared by reversible addition fragmentation chain transfer (RAFT) polymerization as dispersant. Then, the resulting dispersion is engaged in a radical emulsion polymerization process whereby a hydrophobic organic monomer (styrene and butyl acrylate) is polymerized to form the shell of the hybrid nanoparticle. This method is extremely versatile, allowing the preparation of a variety of nanocomposites with metal oxides (alumina, rutile, anatase, barium titanate, zirconia, copper oxide), metals (Mo, Zn), and even inorganic nitrides $\left(\mathrm{Si}_{3} \mathrm{~N}_{4}\right)$.

Copyright (C) 2008 J.-C. Daigle and J. P. Claverie. This is an open access article distributed under the Creative Commons Attribution License, which permits unrestricted use, distribution, and reproduction in any medium, provided the original work is properly cited.

\section{INTRODUCTION}

The preparation of core-shell nanoparticles where the core of the particle is inorganic and the shell is an organic polymer has been the object of a profusion of recent publications $[1,2]$. Indeed, the resulting nanocomposites find wide applications in the field of coatings and adhesives [3,4], optics [5], electronics [6], biomedical [7] and environmental applications [8]. When the core-shell nanoparticle is suspended in water, the equilibrium arrangement of the inorganic phase and the organic polymer mainly depends on the interfacial energies of the mineral/water, polymer/water, and mineral/polymer interfaces. In most cases, the mineral surface being quite polar, its encapsulation by a hydrophobic polymer is not thermodynamically favorable. Thus, it is not surprising that instead of the desirable core-shell arrangement, a myriad of other morphologies is usually observed for these nanocomposites, such as occluded, half-moon, hairy, strawberry-like, and so on $[1,2]$.

There exist two general routes for the preparation of core-shell nanocomposites. The first one relies on the selfassembly of the inorganic nanoparticle with a preformed polymer. For example, layer-by-layer (LbL) deposition of polyelectrolytes [9] and heterocoagulation of positively charged latex [10] onto negatively charged inorganic particles can be used to prepare core-shell type nanocomposites. The second one is based on the direct formation of the polymer in the presence of the mineral nanoparticle. Usually, an emulsion polymerization process is used, because of its robustness, its versatility, and its ability to yield polymer nanoparticles (latexes) with sizes ranging from $10 \mathrm{~nm}$ to 1 micron [11]. Yet, due to the lack of compatibility between the polymer and the inorganic surface, the encapsulation succeeds if the inorganic nanoparticle is treated beforehand to make it more organic-like, for example by adsorption of a surfactant, resulting in the "hydrophobization" of the inorganic surface $[12,13]$. However, this strategy is often plagued by a loss of colloidal stability (aggregation of the inorganic particles). As a means to circumvent this problem, it is possible to use a miniemulsion polymerization process whereby the surfactant-treated inorganic particles are dispersed into stable nanometric droplets of an organic liquid monomer which is later polymerized, to successfully form hybrid nanocomposites [14-16]. Another route consists in forcing the inclusion of the inorganic nanoparticle into the organic shell by tethering it with covalent links. Numerous 


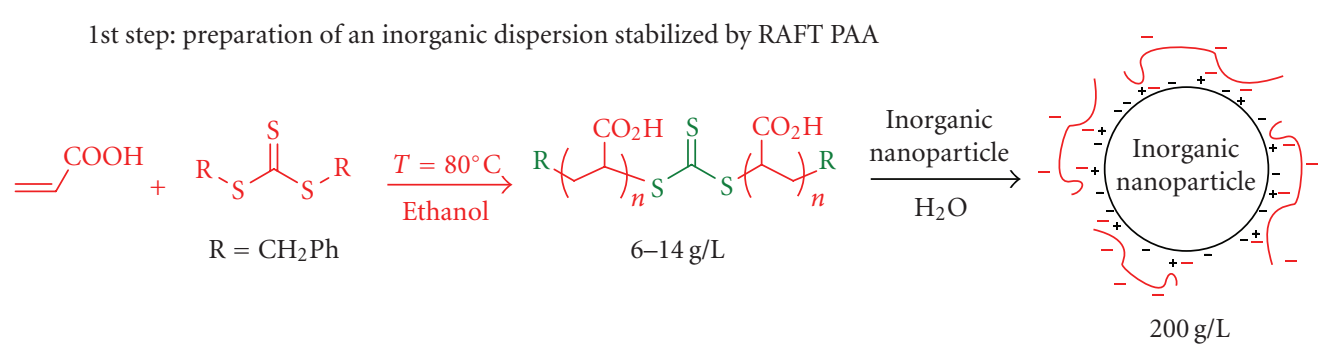

2nd step: encapsulation by emulsion polymerization of styrene
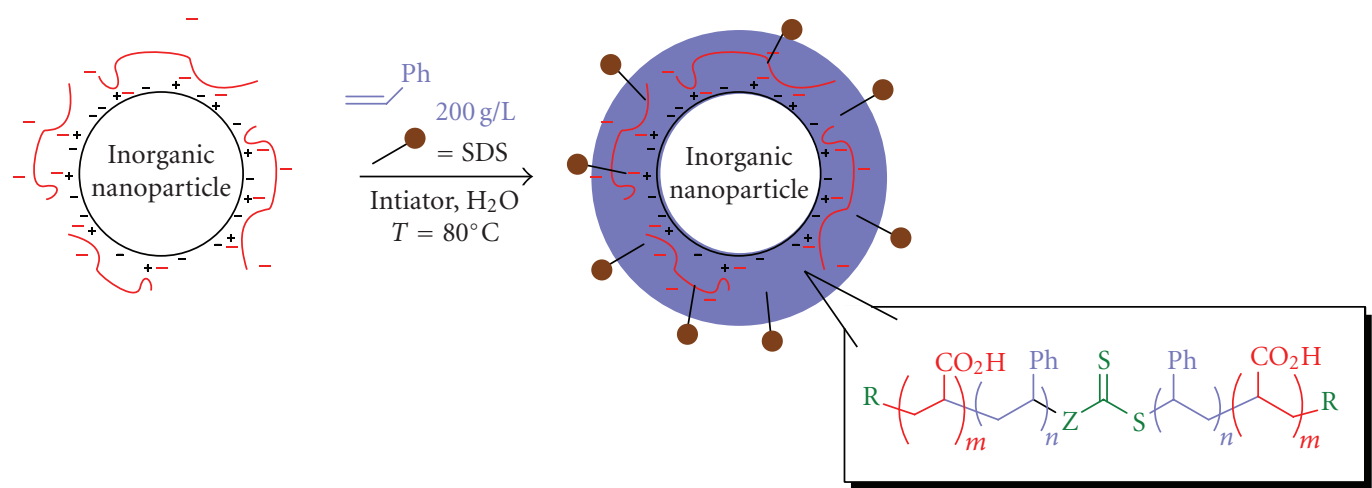

Scheme 1: Encapsulation of an inorganic nanoparticle dispersed in water by PAA (step 1) to form hybrid core-shell nanoparticles (step 2). The organic shell is formed of polystyrene (95\%) and PAA (5\%).

variants have been used, for example, by chemically preparing the mineral surface with reacting groups (such as reactive monomers [17], radical initiators [18], and promoters for controlled radical polymerization [19]). Although often successful, this strategy requires a functionalization step which must be adapted to each nanoparticle (diazonium decomposition on graphite and cobalt [20], silane [21], and titanate [22] coupling agents on silica and alumina, thiols on gold [23], etc.). It is clear that there exists a profusion of methods for the preparation of the core-shell inorganic-organic hybrid nanoparticles, yet all of those have a restricted scope, either being limited to a small set of inorganic particles or to a limited number of polymers and polymer thicknesses (as in the case of LbL deposition). For the sake of completion, it is also important to mention that concomitantly to this work $[24,25]$, the group of Hawkett [26-28] has recently described a similar approach based on an interfacial polymerization process used to encapsulate a variety of inorganic particles.

We here report a general method to synthesize hybrid core-shell nanoparticles. To our surprise, this method proved to be successful for all types of inorganic compounds we tried, being metal oxides, metals, or even metal nitrides. The mineral nanoparticles are first dispersed into water with the aid of a dispersant prepared by controlled radical polymerization (Scheme 1). The shell is then formed upon the addition of organic monomers via an emulsion polymerization process. The key feature of this encapsulation method is the use of a living/controlled dispersant which is reactivated during the polymerization of the shell, thus leading to the formation of block copolymers. The shell is covalently tethered to the dispersant, which is adsorbed onto the inorganic nanoparticle surface.

In comparison to existing methods of nanoencapsulation, the route we propose does not require either modification or optimization when one changes the nature of the inorganic nanoparticles. Importantly, the commercial inorganic nanoparticles do not need to undergo a specific chemical surface treatment before use. The preparation of the composite is exclusively performed in water, thus avoiding the manipulation of organic solvents and bypassing solvent transfer or solvent exchange steps which are often tedious. Last, this method is easily scalable, as we were able to target dispersions containing as much as 30\% in weight of composite with no loss of colloidal stability (no aggregation).

\section{RESULTS AND DISCUSSION}

\subsection{RAFT poly(acrylic acid) as dispersant}

The chosen dispersant for this work is a poly(acrylic acid) (PAA) homopolymer prepared by reversible addition chain transfer (RAFT) polymerization, using trithiobenzyl carbonate (TBC) as RAFT agent (Scheme 1). Based on the pioneering work of Rizzardo on RAFT [29], we have shown in the past that this RAFT agent is suitable to control the polymerization of acrylic acid (AA), yielding PAAs which have a low polydispersity $[30,31]$. Using the analytical methods detailed in [31], it was found that PAA used here has a degree of polymerization of 86 (43 units in average on each 
TABLE 1: Characteristics of the inorganic nanoparticles with a polystyrene shell.

\begin{tabular}{|c|c|c|c|c|c|c|c|c|c|}
\hline Inorganic & S.C. $(\%)^{[a]}$ & core $^{[\mathrm{b}]}$ & $\begin{array}{l}\text { Compos } \\
\text { shell }^{[\mathrm{b}]}\end{array}$ & $\begin{array}{l}\text { n }(\text { wt \%) } \\
\text { core }^{[c]}\end{array}$ & shell ${ }^{[c]}$ & $\mathrm{Dp}^{[\mathrm{d}]}(\mathrm{nm})$ & $\sigma^{[\mathrm{e}]}(\mathrm{nm})$ & shell $^{[\mathrm{f}]}(\mathrm{nm})$ & Slope $e^{[g]}$ \\
\hline Anatase & 9.1 & 54 & 46 & 48 & 52 & 85 & 12 & 18 & $\mathrm{nd}^{[\mathrm{h}]}$ \\
\hline Rutile & 10.7 & 38 & 62 & 72 & 28 & 93 & 27 & 13 & 11 \\
\hline $\mathrm{TiO}_{2}$-coated & 15.7 & 57 & 43 & 72 & 28 & 83 & 9 & 11 & 1 \\
\hline $\mathrm{ZrO}_{2}$ & 13.5 & 56 & 44 & 69 & 31 & 91 & 5 & 13 & 1074 \\
\hline $\mathrm{CuO}$ & 14.9 & 33 & 67 & 70 & 30 & 81 & 16 & 14 & 1.5 \\
\hline $\mathrm{BaTiO}_{3}$ & 16.6 & 58 & 41 & 61 & 39 & 93 & 19 & 19 & 65 \\
\hline $\mathrm{Al}_{2} \mathrm{O}_{3}$ & 16.4 & 49 & 51 & 66 & 34 & 108 & 26 & 15 & nd \\
\hline Mo & 14.3 & 56 & 44 & 73 & 27 & 108 & 18 & 22 & $\ll 1$ \\
\hline $\mathrm{Zn}$ & 27.9 & 53 & 47 & 64 & 36 & 182 & 30 & 38 & $\ll 1$ \\
\hline $\mathrm{Si}_{3} \mathrm{~N}_{4}$ & 11.1 & 61 & 39 & 66 & 34 & 155 & 32 & 22 & $\ll 1$ \\
\hline
\end{tabular}

[a] Solid content of the nanocomposite in water, as determined by gravimetric analysis. [b] Determined by TGA analysis. [c] Measured from the volume fractions obtained on electron micrographs. [d] Particle diameter measured on electron micrographs. [e] Half width of the particle diameter distribution. [f] Shell thickness measured on electron micrographs. [g] Slope of the adsorption isotherm (no unit). [h] not determined.

side of the central trithiocarbonate unit), a polydispersity index of 1.15 , and approxemately $73 \%$ of the chains contain a central trithiocarbonate unit, making them a suitable candidate for reinitiating the polymerization.

PAA prepared by RAFT is an excellent dispersant of metal oxide nanoparticles such as kaolin, calcium carbonate (calcite), and titanium dioxide [32,33]. The adsorption is believed to be driven by electrostatics, and in the case of calcite [33], the adsorption of a RAFT polymer was shown to be irreversible and total (no chain in water until saturation is reached). Last, we [34] and others [35, 36] have shown that PAA prepared by RAFT can be extended with other monomers to form amphiphilic block copolymers, making PAA prepared by RAFT an ideal choice for this study.

\subsection{Dispersion of the inorganic nanoparticles}

To evaluate the generality of this encapsulation method, a selection of 10 different nanoparticles was selected for this work, being metal, metal oxides and nitrides, hydrophilic (e.g., $\mathrm{Al}_{2} \mathrm{O}_{3}$ ), or hydrophobic (e.g., $\mathrm{Si}_{3} \mathrm{~N}_{4}$ ). Silica [37], gold [23], and carbon black [38] nanoparticles were not selected because very efficient and elegant methods have already been developed for those nanoparticles. Before embarking upon a description of the encapsulation process, a few observations relative to the nature of the surface of the nanoparticles can be made. Coated- $\mathrm{TiO}_{2}$ nanoparticles are coated by a thin layer of alumina and are commonly used in the pigment industry, in contrast to anatase and rutile which are often used for their semiconductor and photocatalytic properties. No effort has been made to treat the commercial nanoparticles used in this study before use; therefore, the metallic nanoparticles (Mo and $\mathrm{Zn}$ ) may be passivated by a thin layer of oxide.

The adsorption isotherms of PAA onto commercial inorganic nanoparticles (Figure 1 and Table 1 ) indicate that the adsorption of PAA is, as expected, strongly dependent upon the mineral surface. The isotherms were built by separating the nanoparticles from the continuous phase by centrifugation, and analyzing the supernatant for the amount of PAA free, using the method described in [39]. A precise adsorption isotherm could not be built for anatase and $\mathrm{Al}_{2} \mathrm{O}_{3}$ because the serum is always turbid due to residual amounts of small particles in suspension. The amount of PAA adsorbed onto $\mathrm{Zn}$, Mo, and $\mathrm{Si}_{3} \mathrm{~N}_{4}$ nanoparticles was found to be below measurable levels. This observation may be explained by the absence of electrostatic interactions in between the negatively charged PAA and the neutral surfaces of $\mathrm{Si}_{3} \mathrm{~N}_{4}, \mathrm{Zn}$. and Mo (assuming no passivation for the two latter cases). In the case of $\mathrm{BaTiO}_{3}$ and $\mathrm{ZrO}_{2}$, all the PAA is adsorbed until the surface is saturated. On the contrary, for other inorganic particles $\left(\mathrm{CuO}\right.$, rutile, coated $\left.\mathrm{TiO}_{2}\right)$, the adsorption isotherm corresponds to a typical Langmuir isotherm, showing a partitioning between free and bound PAA. The affinity of PAA for the mineral surface can be quantified by the slope of the adsorption isotherm at low concentrations (Figure 1 and Table 1) and ranks as follows: $\mathrm{ZrO}_{2}>\mathrm{BaTiO}_{3}>$ rutile $>\mathrm{CuO}>$ coated $-\mathrm{TiO}_{2}>$ $\mathrm{Zn} \sim \mathrm{Mo} \sim \mathrm{Si}_{3} \mathrm{~N}_{4}$. In our experiments, $\mathrm{pH}$ was not controlled by a buffer, and measured $\mathrm{pH}$ was comprised between 4 and 7. Influence of $\mathrm{pH}$ and resulting $\zeta$ potential on the behavior of a dispersant are well documented in literature [40]. At higher $\mathrm{pH}$ values, not only the adsorption of PAA on $\mathrm{TiO}_{2}$ nanoparticles becomes unfavorable [28], but also significant hydrolysis of the chain transfer agent occurs [31].

The plateau levels in the adsorption isotherm correspond to the saturation of the surface by PAA. These levels are evidently related to the surface areas of the nanoparticles, and therefore their sizes. The commercial nanoparticles used here come with various size distributions, and, accordingly, the position of the saturation plateau varies greatly. For $\mathrm{ZrO}_{2}$ and $\mathrm{BaTiO}_{3}$ nanoparticles which exhibit a strong binding isotherm, the amount of PAA used for dispersing them prior encapsulation was equal to 1.3 the saturation amount (100\% adsorbed and 30\% free). For the other nanoparticles, an arbitrary amount of $10 \mathrm{~g} / \mathrm{kg}$ of dispersion was used, since, whatever the amount used, PAA is partitioned between free and adsorbed. With this quantity 


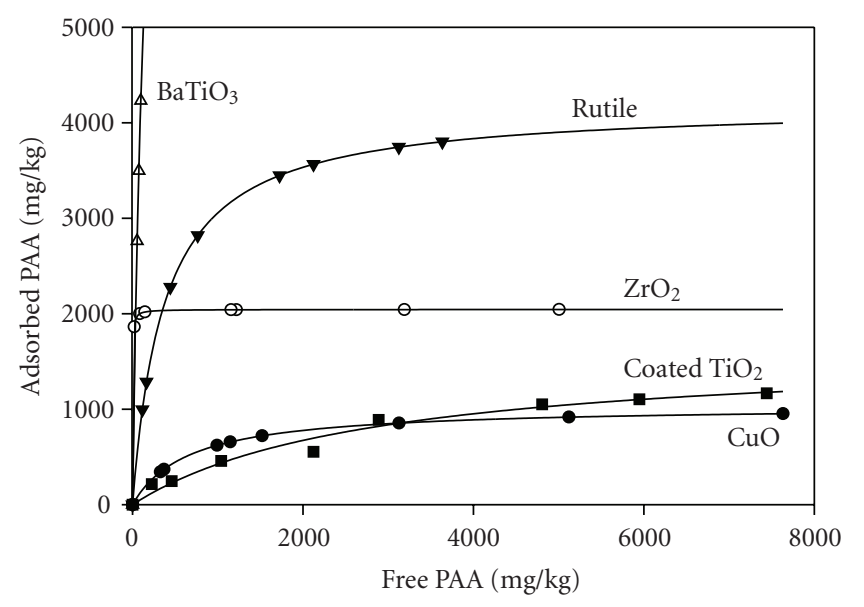

FIgURE 1: Adsorption isotherm of PAA on several inorganic nanoparticles ([inorganic] $=200 \mathrm{~g} / \mathrm{L}$ in water), determined using the method described in [39]. The curves are the best fit of the experimental points with the function $f(x)=K x /(1+a x)$, which corresponds to a theoretical Langmuir isotherm curve.

of PAA, the nanoparticles remain dispersed in water for several hours, which is sufficient to initiate the second step of the synthesis (encapsulation by an hydrophobic polymer). Lower amounts often result in the rapid formation of aggregates, as shown by dynamic light scattering, which ultimately does not lead to the formation of core-shell hybrid nanoparticles. Importantly, notwithstanding the position of the saturation plateau, free (i.e., not adsorbed) PAA is present in the dispersion, either because an amount greater than saturation is used or because the adsorption isotherm shows a partitioning between free and adsorbed PAA. This point will be further discussed in the section on the mechanism of encapsulation.

\subsection{Encapsulation of the inorganic nanoparticles by a polymer}

Once the inorganic nanoparticles are dispersed in water, an organic monomer (styrene) is added (step 2, Scheme 1), in the presence of a nonoxidizing water-soluble initiator $\left(4,4^{\prime}\right.$ azobis-4-cyanovaleric acid) and a small amount of surfactant (sodium dodecylsulfate, SDS) which is necessary to prevent the final core-shell nanoparticles from aggregating. Oxidizing initiators are known to potentially decompose the trithiocarbonate [41]. The amount of initiator added is kept below 20\% the amount of trithiocarbonate units present in the PAA, so as to limit the number of homopolystyrene chains and to favor the formation of triblock copolymers PS-PAA-PS [33]. However, no attempt was made to check whether all chains were blocked because the amount of polystyrene (PS, 3.6g) formed during this step is greatly superior to the amount of PAA introduced as dispersant $(100 \mathrm{mg})$. The fraction of polymer soluble in THF for experiment $3\left(\mathrm{TiO}_{2}\right.$-PS $)$ had a broad molecular weight distribution as measured by GPC $\left(M_{n}=24,500 \mathrm{~g} / \mathrm{mol}\right.$, PDI $=2.2$ ), which indicates that control on MWD is not very effective under these conditions. Numerous reasons can be evoked to explain the loss of control: low efficiency of reinitiating the PAA block with styrene due to a significant mismatch of polarity [42], partial decomposition of the RAFT agent [31], trithiocarbonate RAFT agent not very efficient at controlling the polymerization of styrene [29]. This work does not aim at using controlled radical polymerization techniques for the sake of controlling the MWD but it takes advantage of the RAFT technique to reinitiate a dormant oligomer (PAA dispersant) which has a high chain-end reactivity. Importantly, the formation of core-shell structures is unsuccessful if one uses a conventional (dead) PAA, pointing out that block copolymers must be formed during this experiment, probably in conjunction with an unquantified amount of PS homopolymer chains.

\subsection{Characterization of the core-shell nanoparticles}

The characteristics of the resulting nanoparticles are consigned in Table 1. The solid content of the dispersion is typically around $10 \mathrm{wt} \%$, but can be as high as $25 \mathrm{wt} \%$, which is an indication of the colloidal stability of this system. The global composition of the nanoparticles has been assessed by TGA analysis: in average the composite is constituted by $50 \mathrm{wt} \%$ of inorganic matter and $50 \mathrm{wt} \%$ of polymer. This composition corresponds precisely to the ratio of the weight of mineral nanoparticles to organic monomer prior polymerization, thus indicating that the conversion of monomer to polymer is high.

The morphology of the nanocomposite has also been assessed by TEM (Figure 2). The inorganic nanoparticle is encapsulated by a shell of polymer with a thickness comprised between 10 and $40 \mathrm{~nm}$ (Table 1), with no aggregates and no partially encapsulated inorganic nanoparticles. The particle size distribution (Table 1) was calculated from the surface areas obtained in a digital treatment of binarized pictures. The thickness of the shell was directly measured on the micrographs (average of more than 200 measurements, but several measurements per particle). From the thickness of the shell and the diameter of the nanoparticle, it is possible to assess the volume fractions of the core and shell assuming a spherical shape, which can in turn be used to calculate the corresponding weight fractions, knowing the respective density of the inorganic particle and of the polymer (density $=1$ ). In certain cases, the composition of the nanocomposite is identical to the one measured by TGA (anatase, $\mathrm{BaTiO}_{3}, \mathrm{Zn}$ ), but in other cases, the relative amount of shell is lower than expected. For the latter case, this discrepancy can be attributed to the presence of polymer nanoparticles devoid of inorganic core, which can clearly be identified in some of the micrographs ( $\mathrm{x}$ in Figure 2(b), 2(e), and $2(\mathrm{j})$ ). Once the polymerization is triggered, the PAA is either adsorbed at the particle surface or free in water, since an amount greater than saturation is used. Upon extension by styrene, the copolymer becomes amphiphilic and behaves as an in situ surfactant which is able to nucleate a new crop of "empty" particles.

The encapsulation method is quite general and is also efficient to form polymeric shells of lower $\mathrm{Tg}$, for example, 


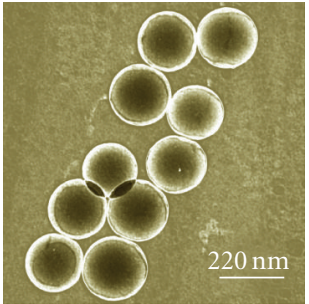

(a)

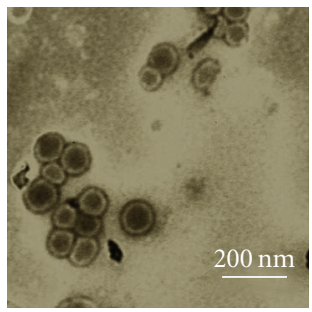

(c)

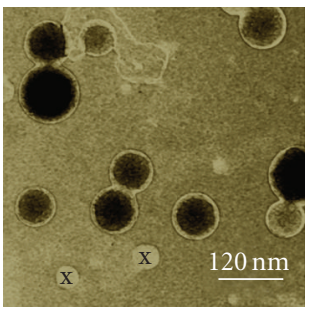

(e)

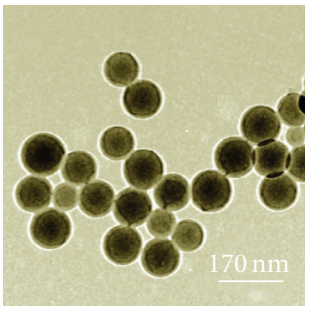

(g)

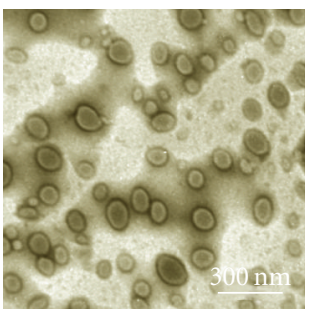

(i)

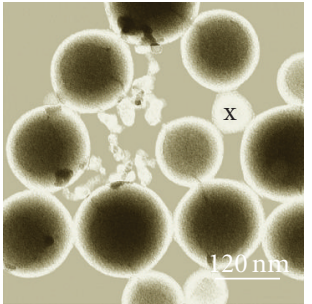

(b)

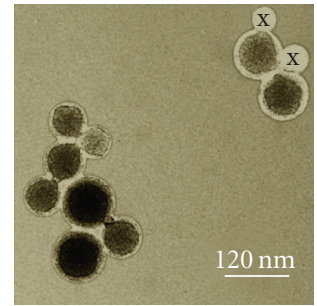

(d)

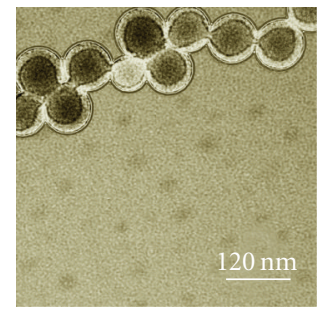

(f)

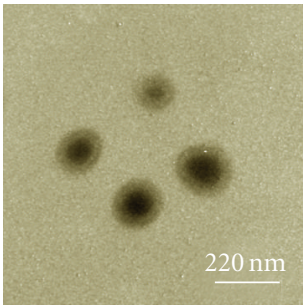

(h)

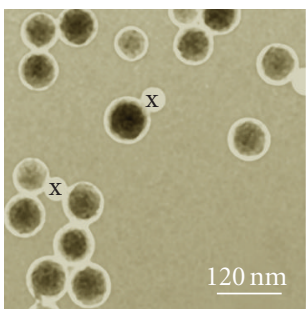

(j)
FIgURE 2: Transmission electron micrograph of the inorganic particles encapsulated in a polystyrene shell. (a) $\mathrm{BaTiO}_{3}$, bar = $220 \mathrm{~nm}$; (b) $\mathrm{Si}_{3} \mathrm{~N}_{4}$, bar $=120 \mathrm{~nm}$; (c) anatase, bar = $200 \mathrm{~nm}$; (d) $\mathrm{TiO}_{2}$ coated, bar $=120 \mathrm{~nm}$; (e) rutile, bar $=120 \mathrm{~nm}$; (f) $\mathrm{ZrO}_{2}$, bar = $120 \mathrm{~nm}$; (g) $\mathrm{Al}_{2} \mathrm{O}_{3}$, bar = $170 \mathrm{~nm}$; (h) $\mathrm{Zn}$, bar $=220 \mathrm{~m}$; (i) Mo, bar $=300 \mathrm{~nm}$; (j) $\mathrm{CuO}$, bar $=120 \mathrm{~nm}$.

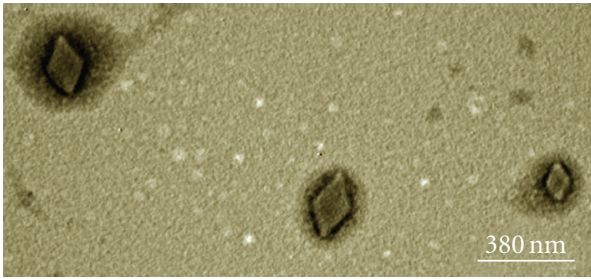

(a)

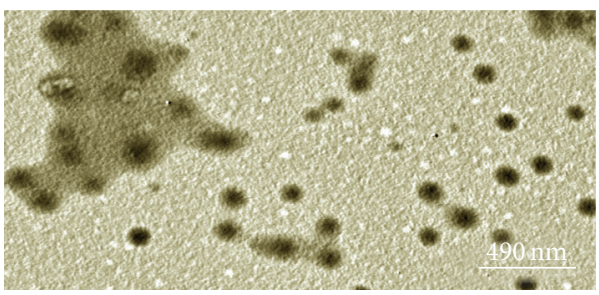

(b)

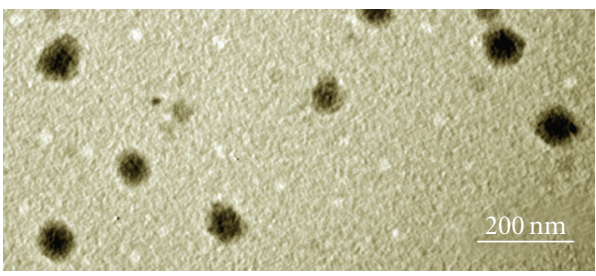

(c)

FIgURE 3: Transmission electron micrograph of the inorganic particles encapsulated in a poly(styrene-co-butyl acrylate) shell. (a) Rutile, bar $=380 \mathrm{~nm}$; (b) $\mathrm{TiO}_{2}$ coated, bar $=490 \mathrm{~nm}$; (c) anatase, bar $=200 \mathrm{~nm}$.

shells constituted of a copolymer of n-butyl acrylate and styrene $\left(50: 50 \mathrm{w}: \mathrm{w}\right.$, calculated $\left.\mathrm{Tg}=7^{\circ} \mathrm{C}\right)$. In this case, the electron micrographs (Figure 3) also clearly indicate the formation of core-shell nanoparticles but the shell is not spherical, because in the dry state (for TEM analysis) the polymer can spread on the surface of the grid. In certain cases, a continuous film is formed due to the coalescence of several particles together (Figure 3(b) top left). It is interesting to note that the inorganic nanoparticles in the film are not touching each other, which is a legacy of the fact that they were encapsulated in a core-shell structure before merging together into a continuous film.

\subsection{Encapsulation mechanism}

Why is this encapsulation method so general? Contrarily to what was observed in the case of $\mathrm{CaCO}_{3}$ [32], the adsorption of PAA onto the surface of inorganic nanoparticles is not always irreversible, but ranges from very strong to weak, as shown by the very diverse adsorption isotherms (Figure 1). We envisage below two limiting cases, depending on the adsorption behavior of PAA on the particle surface. Before presenting these two cases, it is important to stress that the adsorption isotherm corresponds to the partitioning between the free and adsorbed PAA which itself can be 
viewed as the ratio of the adsorption to desorption rate constants. In a first approximation, the adsorption rate constant does not depend on the inorganic surface (but only on the diffusion coefficient of the PAA). Therefore, the partition factor is inversely proportional to desorption rate constant, hence, proportional to the time the PAA is staying at the surface.

When the polymer is strongly adsorbed, there is no exchange (or a very slow exchange) between the adsorbed and the free polymer. Once the polymerization is triggered, the monomer has no other choice than inserting on the adsorbed polymer and on the free polymer. The growing adsorbed polymer, becoming more and more hydrophobic, will constitute the shell, whereas the free polymer eventually becomes amphiphilic and can either generate a crop of new particles (nucleation event in emulsion polymerization) or adsorb onto the surface offered by the inorganic nanoparticles now partially "hydrophobized." Statistically, this latter event is favored because the polymerization is performed in the presence of a large amount of inorganic nanoparticles (20 wt $\%)$, representing a huge surface area. Thus, most of the polymer serves to the formation of a shell, but there is a risk that a few polymer-only particles are created.

When there is a rapid exchange between the adsorbed and free polymer (weak adsorption), each time a monomer unit is inserted, the copolymer PS-PAA-PS becomes more hydrophobic and the exchange time scale between the adsorbed and free states becomes slower. At one point, the exchange becomes so slow (no more solubility of the polymer in water) that the situation becomes identical to the case of a strong adsorption: the copolymer is frozen at the inorganic surface.

It could be argued that this method would be most successful if the amount of dispersant would always be below saturation, so as to avoid the presence of free PAA in the aqueous phase. However, this would only be possible for those particles showing a strong irreversible isotherm $\left(\mathrm{BaTiO}_{3}, \mathrm{ZrO}_{2}\right.$, in this study), which would limit the versatility of the method. Since the precise determination of the adsorption isotherm can be quite tedious (e.g., in the case of $\mathrm{CaCO}_{3}$, the $\mathrm{CO}_{2}$ partial pressure must be controlled [31]), it is preferable to use a method which is efficient notwithstanding the ratio of PAA versus saturation amount, as long as the resulting dispersion exhibits sufficient colloidal stability to engage the encapsulation step. In this study, we found that PAA concentrations of 6-14 g/L are optimal for inorganic nanoparticle concentrations of $200 \mathrm{~g} / \mathrm{L}$. However, the PAA concentration will have to be adjusted by trial and error when the ionic strength of the aqueous phase is changed, and if the molecular weight and nature of the dispersant are changed.

In this study, SDS surfactant was added to the emulsion recipe to provide a better stabilization of the latex particles. The SDS concentration $(1.2 \mathrm{~g} / \mathrm{L})$ was kept well below $\mathrm{cmc}$ $\left(2.4 \mathrm{~g} / \mathrm{L}\right.$ at $\left.25^{\circ} \mathrm{C}\right)$ in order to minimize secondary nucleation. Indeed, no secondary nucleation occurs for anatase, $\mathrm{BaTiO}_{3}$, and $\mathrm{Zn}$, seemingly indicating that secondary nucleation occurs only when PAA is weakly adsorbed, and it is not due to the presence of SDS. The presence of a small amount of particles generated by secondary nucleation has also been reported by Nguyen et al. [28], who have been able to encapsulate several pigments using a macroRAFT agent based on a random amphiphilic copolymer containing butyl acrylate and acrylic acid. In their work, no surfactant was used, reinforcing the idea that secondary nucleation is not due to the presence of surfactant.

\section{CONCLUSION}

In this preliminary report, a remarkably simple technique which is convenient for the preparation of a wide range of inorganic-organic hybrid nanoparticles has been unraveled. The method is successful for both hydrophilic nanoparticles (e.g., alumina) or hydrophobic ones $\left(\mathrm{Si}_{3} \mathrm{~N}_{4}\right.$, e.g.). This uncomplicated and rapid method has been evaluated in an aqueous only environment, which is advantageous for many applications. Yet, in a future work, the possibility to adapt this strategy to an organic solvent will be assessed, starting with a living/controlled dispersant in organic medium and extending the chains with a monomer such as the copolymer does not exhibit any solubility in the organic solvent. Another avenue which needs to be explored in the future is the possibility to use other controlled polymerization techniques. If the mechanism proposed above for the formation of the hybrid particles is exact, then, there should be no limitations as to the choice of the controlled mechanism. Finally, although the scope of the radical emulsion polymerization process is very broad, it often leads to polymers with illdefined microstructures which are not easily functionalized. Conceptually, the method described here, which can be viewed as a method of "grafting from" the dispersant by a reactive process, could be extended to a variety other polymerization techniques (ROMP, late transition metal catalysis, ROP, etc.) thus opening the route to an easy path for the formation of functionalized core-shell nanoparticles.

\section{EXPERIMENTAL}

\subsection{Materials and analytical methods}

Nanoparticles of $\mathrm{TiO}_{2}$ rutile, $\mathrm{TiO}_{2}$ anatase, $\mathrm{ZrO}_{2}$, and $\mathrm{CuO}$ were purchased at MTI corporation (2700 Rydin Rd, Unit D, Richmond, Calif., USA). The $\mathrm{TiO}_{2}$ nanoparticles, from Dupont de Nemours, were $95 \% \mathrm{TiO}_{2}$-coated by a thin layer of alumina. All other chemicals were obtained at Aldrich Company. TGA measurements were obtained using the SII Exstan 6000 TG/DTA 6200 of Seiko Instruments Inc. The sample was heated at $10^{\circ} \mathrm{C} / \mathrm{min}$ with a plateau at $250^{\circ} \mathrm{C}$ for 10 minutes, and then heated at $10^{\circ} \mathrm{C} / \mathrm{min}$ with a plateau at $450^{\circ} \mathrm{C}$ for 15 minutes, then heated to $900^{\circ} \mathrm{C}$. The amount of polymer corresponds to the quantity degraded between 250 and $450^{\circ} \mathrm{C}$, and the amount of inorganic matter to the remaining solid. Below $250^{\circ} \mathrm{C}$, water is evaporated. TEM micrographs were obtained with a Tecnai 12 Biotwin microscope equipped with a W source (80 and $120 \mathrm{kV}$ ) and a 13 Mpixel Morada camera, except for the $\mathrm{Si}_{3} \mathrm{~N}_{4}$ nanocomposite for which a $1 \mathrm{Mpixel} \mathrm{Gatan} \mathrm{camera} \mathrm{was} \mathrm{used.}$ The samples were obtained by depositing a dilute sample 
on a gold grid coated with a formvar film. No contrast agent was used. The images were analyzed using image software developed by the NIH (http://rsb.info.nih.gov/ij/). Low voltage $(80 \mathrm{kV})$ favored contrast between the core and the shell, but higher voltage $(120 \mathrm{kV})$ favored resolution at high magnification. The molecular weight distribution of the polymer generated in experiment 3, relative to polystyrene standards, was determined on a Waters GPC equipped with an RI detector and a set of Polymer Laboratories Mixed C columns. The UV-Vis spectrophotometer was a Cary 50 set at $210 \mathrm{~nm}$.

\subsection{Synthesis of PAA}

PAA synthesis is described in details in [30-34]. The following quantities were used: $402 \mathrm{mg}$ of trithiobenzylcarbonate (TBC, $1.58 \mathrm{mmol}), 10 \mathrm{~mL}$ of acrylic acid (0.132 mol), $48 \mathrm{mg}$ of azobisisobutyronitrile (AIBN, $0.29 \mathrm{mmol}$ ), and refluxing ethanol $40 \mathrm{~mL}$. After 2 hours, the reaction was stopped by placing the mixture in contact to air and adding an inhibitor (BHT). The solution was then concentrated by rotary evaporation until approximately $15 \mathrm{~mL}$ of ethanol were left and the viscous solution was slowly added to $480 \mathrm{~mL}$ of benzene under vigorous stirring. The precipitate was collected and redissolved in the minimum of ethanol. This cycle of dissolution precipitation was repeated four times. The polymer was then dried in vacuo.

\subsection{Typical synthesis of a core-shell nanoparticles}

$4 \mathrm{~g}$ of $\mathrm{ZrO}_{2}, 210 \mathrm{mg}$ of PAA, and $20 \mathrm{~mL}$ of nanopure water $(\rho=18.1 \mathrm{M} \Omega \cdot \mathrm{cm})$ were placed in a $50 \mathrm{~mL}$ beaker immersed in an ice bath. The mixture was stirred with a magnetic stir bar and sonicated during 6 minutes with a sonicator (VibraCell $400 \mathrm{~W}$ ) equipped with a microtip. An off-white dispersion of $\mathrm{ZrO}_{2}$ in water was obtained. In a $250 \mathrm{~mL}$ round-bottom flask, butyl acrylate $(2.0 \mathrm{~mL})$, styrene $(2.0 \mathrm{~mL}), 50 \mathrm{mg}$ of sodium dodecyl sulfate, and $20 \mathrm{~mL}$ of the $\mathrm{ZrO}_{2}$ dispersion were slowly stirred and degassed during 30 minutes by sparging it with argon. The mixture was heated to $80^{\circ} \mathrm{C}$. A solution containing $43 \mathrm{mg}$ of $4,4^{\prime}$-azobis4-cyanovaleric acid, 2 droplets of sodium hydroxide (30\%), and $20.2 \mathrm{~mL}$ of ultrapure water was added continuously over 4 hours $\left(5 \mathrm{~cm}^{3} /\right.$ hour $)$. The role of the sodium hydroxide was to facilitate the dissolution of the initiator (carboxylic acid). When the addition was over, the mixture was left at $80^{\circ} \mathrm{C}$ for one hour in order to complete the reaction.

\subsection{Determination of the adsorption isotherm [39]}

A series of stock solutions of PAA in water $(50-200 \mathrm{mg} / \mathrm{L})$ was prepared and used to build a calibration curve by measurement of the absorption at $210 \mathrm{~nm}$. $\mathrm{TiO}_{2}(2 \mathrm{~g})$ were added to $10 \mathrm{~mL}$ of water (concentration $200 \mathrm{~g} / \mathrm{L}$ identical to the one used for the encapsulation) containing a known amount of PAA. The mixture was sonicated for 3 minutes and left for 2 hours. The nanoparticles were separated from the serum by centrifiguation ( 30 minutes at $4000 \mathrm{rpm}$ ). The concentration of PAA in the serum was then analyzed by UVVIS.

\section{REFERENCES}

[1] E. Bourgeat-Lami, "Organic/inorganic nanocomposite colloids," in Encyclopedia of Nanoscience and Nanotechnology, H. S. Nalwa, Ed., vol. 8, pp. 305-332, American Scientific, Stevenson Ranch, Calif, USA, 2004.

[2] E. Bourgeat-Lami, "Hybrid organic/inorganic particles," in Hybrid Materials, Synthesis, Characterization and Applications, G. Kickelbick, Ed., pp. 87-148, Wiley-VCH, Weinheim, Germany, 2007.

[3] G. Wagner, "Coatings with functionality: the European Coatings Conference "Smart Coatings" in Berlin," European Coatings Journal, no. 7-8, pp. 44-76, 2002.

[4] A. M. van Herk and A. L. German, "Microencapsulated pigments and fillers," in Microspheres, Microcapsules \& Liposomes, A. Guyot, Ed., vol. 1, pp. 457-486, Citus, London, UK, 1999.

[5] L. L. Beecroft and C. K. Ober, "Nanocomposite materials for optical applications," Chemistry of Materials, vol. 9, no. 6, pp. 1302-1317, 1997.

[6] K. Rajeshwar, N. R. de Tacconi, and C. R. Chenthamarakshan, "Semiconductor-based composite materials: preparation, properties, and performance," Chemistry of Materials, vol. 13, no. 9, pp. 2765-2782, 2001.

[7] M. Boissiere, J. Allouche, and T. Coradin, "Biomimetic biopolymer/silica capsules for biomedical applications," in Handbook of Biomineralization: Biomimetic and Bioinspired Chemistry, E. Bäuerlein, P. Behrens, and M. Epple, Eds., pp. 353-369, Wiley-VCH, New York, NY, USA, 2007.

[8] J. Choi, O. Kim, and S.-Y. Kwak, "Suppression of dioxin emission in co-incineration of poly(vinyl chloride) with $\mathrm{TiO}_{2}$-encapsulating polystyrene," Environmental Science \& Technology, vol. 41, no. 16, pp. 5833-5838, 2007.

[9] E. Donath, G. B. Sukhorukov, F. Caruso, S. A. Davis, and H. Möhwald, "Novel hollow polymer shells by colloidtemplated assembly of polyelectrolytes," Angewandte Chemie International Edition, vol. 37, no. 16, pp. 2201-2205, 1998.

[10] K. Csoban and E. Pefferkorn, "Perikinetic aggregation induced by chromium hydrolytic polymer and sol," Journal of Colloid and Interface Science, vol. 205, no. 2, pp. 516-527, 1998.

[11] A. M. V. Herk, Chemistry and Technology of Emulsion Polymerisation, Blackwell, Oxford, UK, 2005.

[12] M. Hasegawa, K. Arai, and S. Saito, "Uniform encapsulation of fine inorganic powder with soapless emulsion polymerization," Journal of Polymer Science Part A, vol. 25, no. 11, pp. 3117-3125, 1987.

[13] D.-G. Yu, J. H. An, J. Y. Bae, S. D. Ahn, S.-Y. Kang, and K. S. Suh, "Preparation of titanium dioxide/poly(methyl methacrylate-co-n-butyl acrylate-co-methacrylic acid) hybrid composite particles via emulsion polymerization," Journal of Applied Polymer Science, vol. 97, no. 1, pp. 72-79, 2005.

[14] B. Erdem, E. D. Sudol, V. L. Dimonie, and M. S. El-Aasser, "Encapsulation of inorganic particles via miniemulsion polymerization. III. Characterization of encapsulation," Journal of Polymer Science Part A, vol. 38, no. 24, pp. 4441-4450, 2000.

[15] B. Erdem, E. D. Sudol, V. L. Dimonie, and M. S. El-Aasser, "Encapsulation of inorganic particles via miniemulsion polymerization. I. Dispersion of titanium dioxide particles in organic media using OLOA 370 as stabilizer," Journal of Polymer Science Part A, vol. 38, no. 24, pp. 4419-4430, 2000. 
[16] F. Tiarks, M. Willert, K. Landfester, and M. Antonietti, "The controlled generation of nanosized structures in miniemulsions," Progress in Colloid and Polymer Science, vol. 117, pp. 110-112, 2001.

[17] F. Corcos, E. Bourgeat-Lami, C. Novat, and J. Lang, "Poly(styrene- $b$-ethylene oxide) copolymers as stabilizers for the synthesis of silica-polystyrene core-shell particles," Colloid \& Polymer Science, vol. 277, no. 12, pp. 1142-1151, 1999.

[18] O. Prucker and J. Rühe, "Mechanism of radical chain polymerizations initiated by azo compounds covalently bound to the surface of spherical particles," Macromolecules, vol. 31, no. 3, pp. 602-613, 1998.

[19] T. von Werne and T. E. Patten, "Preparation of structurally well-defined polymer-nanoparticle hybrids with controlled/living radical polymerizations," Journal of the American Chemical Society, vol. 121, no. 32, pp. 7409-7410, 1999.

[20] R. N. Grass, E. K. Athanassiou, and W. J. Stark, "Covalently functionalized cobalt nanoparticles as a platform for magnetic separations in organic synthesis," Angewandte Chemie International Edition, vol. 46, no. 26, pp. 4909-4912, 2007.

[21] E. Bourgeat-Lami, Ph. Espiard, and A. Guyot, "Poly(ethyl acrylate) latexes encapsulating nanoparticles of silica: 1 . Functionalization and dispersion of silica," Polymer, vol. 36, no. 23, pp. 4385-4389, 1995.

[22] A. M. de Oliveira, M. L. C. P. da Silva, G. M. Alves, P. C. de Oliveira, and A. M. dos Santos, "Encapsulation of $\mathrm{TiO}_{2}$ by emulsion polymerization with methyl metacrylate (MMA)," Polymer Bulletin, vol. 55, no. 6, pp. 477-484, 2005.

[23] S. Nuss, H. Böttcher, H. Wurm, and M. L. Hallensleben, "Gold nanoparticles with covalently attached polymer chains," Angewandte Chemie International Edition, vol. 40, no. 21, pp. 4016-4018, 2001.

[24] J. P. Claverie and J. C. Daigle, Patent US60/972, 459, p. 53, 2007.

[25] J. C. Daigle and J. P. Claverie, PMSE Preprints, 98, 685, 2008.

[26] B. S. Hawkett, C. H. Such, and D. Nguyen, "Polymer product and interfacial polymerisation process using raft agent," WO/2007/112503, p. 76, 2007.

[27] B. S. Hawkett, C. H. Such, D. Nguyen, J. M. Farrugia, and O. M. McKinnon, "Surface polymerisation process and polymer product using RAFT agent,” WO/2006/037164, p. 145, 2006.

[28] D. Nguyen, H. S. Zondanos, J. M. Farrugia, A. K. Serelis, C. H. Such, and B. S. Hawkett, "Pigment encapsulation by emulsion polymerization using macro-RAFT copolymers," Langmuir, vol. 24, no. 5, pp. 2140-2150, 2008.

[29] J. Chiefari, Y. K. Chong, F. Ercole, et al., "Living free-radical polymerization by reversible addition-fragmentation chain transfer: the RAFT process," Macromolecules, vol. 31, no. 16, pp. 5559-5562, 1998.

[30] C. Ladavière, N. Dörr, and J. P. Claverie, "Controlled radical polymerization of acrylic acid in protic media," Macromolecules, vol. 34, no. 16, pp. 5370-5372, 2001.

[31] M.-F. Llauro, J. Loiseau, F. Boisson, F. Delolme, C. Ladavière, and J. Claverie, "Unexpected end-groups of poly(acrylic acid) prepared by RAFT polymerization," Journal of Polymer Science Part A, vol. 42, no. 21, pp. 5439-5462, 2004.

[32] J. Loiseau, N. Doërr, J. M. Suau, et al., "Synthesis and characterization of poly(acrylic acid) produced by RAFT polymerization. Application as a very efficient dispersant of $\mathrm{CaCO}_{3}$, Kaolin, and $\mathrm{TiO}_{2}$," Macromolecules, vol. 36, no. 9, pp. 3066-3077, 2003.
[33] J. Loiseau, C. Ladavière, J. M. Suau, and J. Claverie, "Dispersion of calcite by poly(sodium acrylate) prepared by reversible addition-fragmentation chain transfer (RAFT) polymerization," Polymer, vol. 46, no. 19, pp. 8565-8572, 2005.

[34] N. Gaillard, A. Guyot, and J. Claverie, "Block copolymers of acrylic acid and butyl acrylate prepared by reversible additionfragmentation chain transfer polymerization: synthesis, characterization, and use in emulsion polymerization," Journal of Polymer Science Part A, vol. 41, no. 5, pp. 684-698, 2003.

[35] M. Jacquin, P. Muller, G. Lizarraga, C. Bauer, H. Cottet, and O. Théodoly, "Characterization of amphiphilic diblock copolymers synthesized by MADIX polymerization process," Macromolecules, vol. 40, no. 8, pp. 2672-2682, 2007.

[36] C. J. Ferguson, R. J. Hughes, D. Nguyen, et al., "Ab initio emulsion polymerization by RAFT-controlled self-assembly," Macromolecules, vol. 38, no. 6, pp. 2191-2204, 2005.

[37] C. Bartholome, E. Beyou, E. Bourgeat-Lami, P. Chaumont, and N. Zydowicz, "Nitroxide-mediated polymerizations from silica nanoparticle surfaces: "graft from" polymerization of styrene using a triethoxysilyl-terminated alkoxyamine initiator," Macromolecules, vol. 36, no. 21, pp. 7946-7952, 2003.

[38] F. Tiarks, K. Landfester, and M. Antonietti, "Encapsulation of carbon black by miniemulsion polymerization," Macromolecular Chemistry and Physics, vol. 202, no. 1, pp. 51-60, 2001.

[39] M. Huldén and E. Sjöblom, "Adsorption of some common surfactants and polymers on $\mathrm{TiO}_{2}$-pigments," Progress in Colloid and Polymer Science, vol. 82, pp. 28-37, 1990.

[40] H.-H. Wu and K.-C. Hsu, "Effect of $\mathrm{pH}$ on the properties of barium titanate slurries with an aniomic dispersant," Journal of Applied Polymer Science, vol. 101, no. 2, pp. 1082-1088, 2006.

[41] I. Uzulina, S. Kanagasabapathy, and J. Claverie, "Reversible addition fragmentation transfer (RAFT) polymerization in emulsion," Macromolecular Symposia, vol. 150, no. 1, pp. 3338, 2000.

[42] M. Manguian, M. Save, and B. Charleux, "Batch emulsion polymerization of styrene stabilized by a hydrophilic macroRAFT agenta," Macromolecular Rapid Communications, vol. 27, no. 6, pp. 399-404, 2006. 

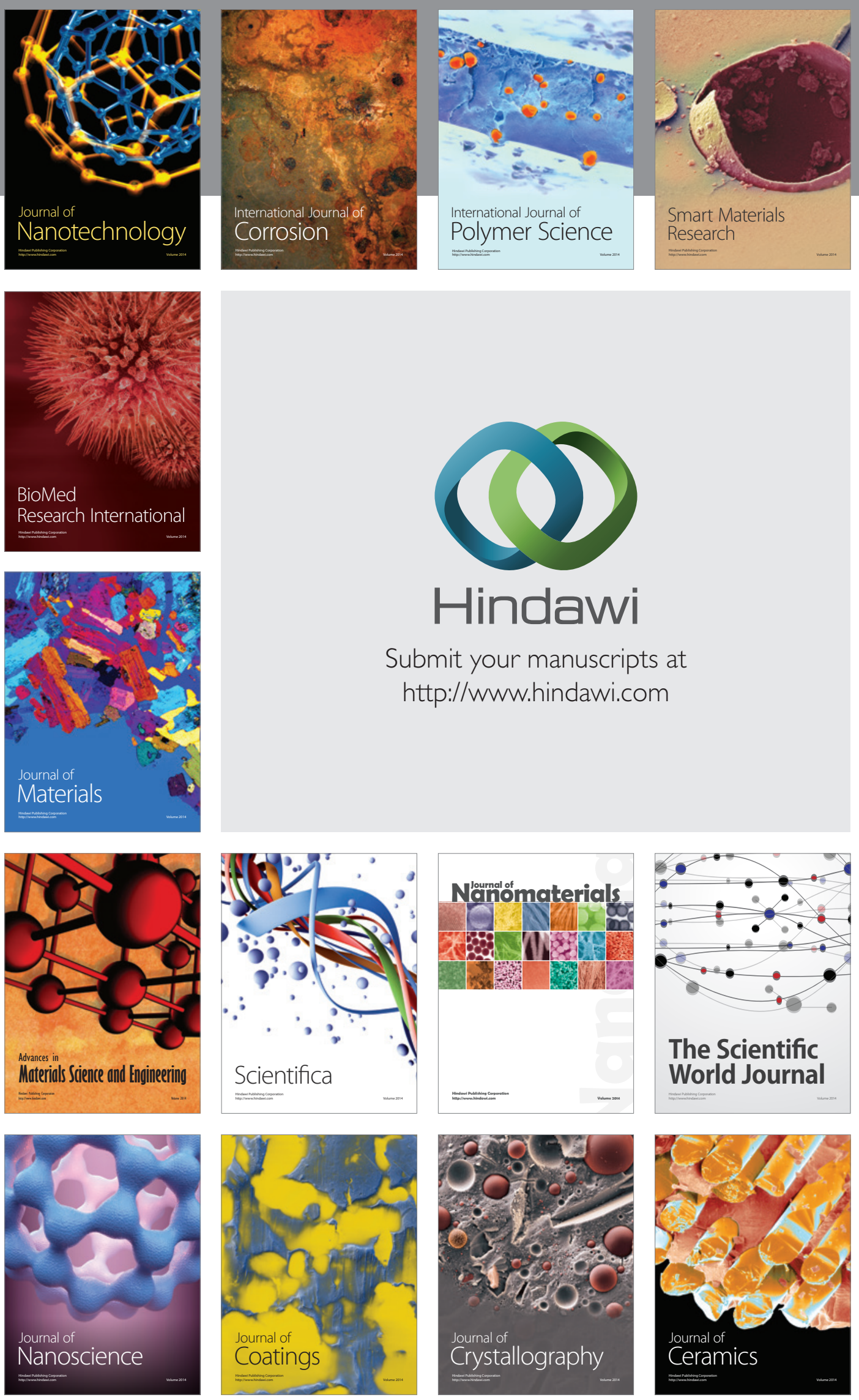

The Scientific World Journal

Submit your manuscripts at

http://www.hindawi.com

\section{World Journal}

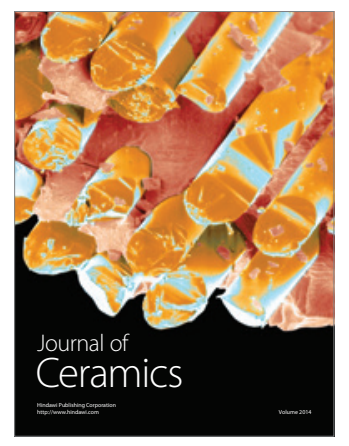

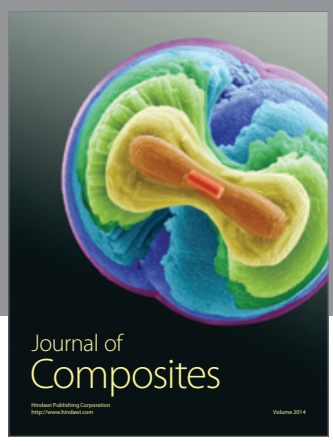
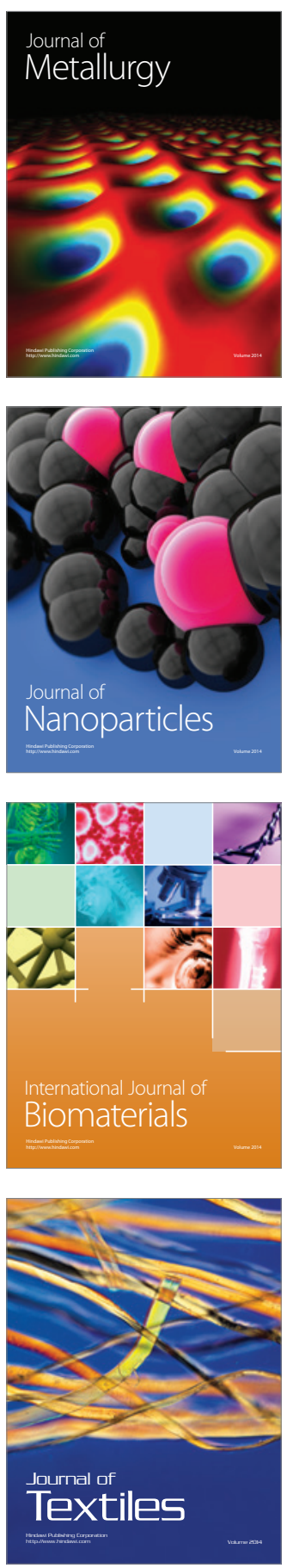